\section{On reduction and regression in the definition of motivation}

\section{ROY A. WISE \\ Concordia University, Montreal, Quebec, Canada}

Blackburn and Pfaus (1988) raise two major concerns about my proposal (Wise, 1987) to define motivation as modulation of stimulus-response effectiveness: (1) After agreeing that other definitions of motivation are circular, they assert that the notion of modulation is also circular; and (2) they argue that my attempt to anchor the notion of modulation to physiology is "reductionistic" and thus resorts to an inappropriate "level" of analysis. The suggestion that physiological analysis is inappropriate is surprising, given the subject matter of this journal; in any case, the definition of modulation is based equally on behavioral and physiological examples.

First, the metaphor of levels of analysis wrongly suggests vertical distinctions between behavioral, physiological, chemical, and physical constructs. For reasons lost to history, the physical sciences are assigned the lowest position, the biological sciences a middle position, and the social sciences the most elevated position in this conceptual hierarchy of analytic levels. We are said to "descend" to physiological or physical analysis, and, by implication, to "rise"' to behavioral, anthropological, or political theory. The metaphor of "levels" of analysis is unfortunate; it serves to rationalize our tendency to restrict ourselves to the arbitrary domain of our narrow specialties, rather than to attempt to develop an appreciation of the teachings of "adjacent" and overlapping disciplines.

Rather than thinking of the scientific enterprise as a vertical hierarchy, we might think of it as a linear (and perhaps horizontal) pursuit of the chain of cause and effect. I am not so much interested in jumping up and down across different levels of analysis as in followingwherever it leads-the linear chain of cause and consequence. If the phenomenon of interest starts with the exposure of an animal to a distal stimulus, such as a water spout, the linear analysis of cause and effect might begin with the visual image of the spout and might end with the act of drinking. For the true behaviorist, these events, coupled with knowledge of the animal's recent drinking history, might offer a sufficiently detailed analysis; for those whose curiosity is not satisfied, the chain of cause and effect leads across the boundaries of the animal, to the retina, the optic nerve, and points in the depth of the brain. Complete scientific understanding of the act of drinking involves answering every question of the form "And what

The author's mailing address is Department of Psychology, Concordia University, 1455 de Maisonneuve Blvd. West, Montreal, P.Q. H3G 1M8, Canada. comes next?" that might be asked about the chain of events between the exposure of the animal to the stimulus and the observation of its swallowing at the drinking spout. (It may also include some events prior to that exposure, such as the conditions that led to the immediate state of the animal's hydration.) Some such events can be classified as "psychological" and distinguished arbitrarily from other events classified as "physiological." But surely it is the chain of events, and not their classification, that concerns us. The metaphor of levels of analysis does not deal with the chain of events, but only the classification of its elements.

The conclusions that derive from the metaphor are clearly nonsense, however. Suppose we were interested in assassinations, and we observed a correlation between the aiming of a gun by the assassin and the falling of the victim some distance away. We might infer that a projectile passes from the gun to the victim, and we might consider this inference to be confirmed by the fact that pieces of lead similar in shape and markings to one found in the victim also were found in the gun. No one has ever observed a bullet in motion, yet we would all be willing to state that we "know" that they travel from gun to target, and that they begin such travel shortly after a puff of smoke appears at the end of the barrel and (less shortly) after a sharp sound emanates from the chamber. Our knowledge of the fact that bullets are projected from guns is based largely on our understanding of how guns work. We understand the principles of the trigger as a lever that cocks and releases a hammer, the hammer as a mass that strikes the sensitive end of the bullet, a bullet as a capsule containing explosive powder, explosion as a process that causes the conversion of solids to gases, gases as forms of matter that take more space than solids (and thus ignition as a process that leads to expansion of matter), the bullet chamber as having an open end through which the bullet can exit and a dead end through which it cannot, the grooves in the barrel as capable of imparting spin to a projectile so that it travels like a spiral pass and not an end-over-end kick. We "understand" all or most of these things about how bullets are sent from guns to targets, and our understanding proceeds linearly, step by step (often with unequal gaps between the steps) from the observable squeezing of the trigger to the observable falling of the victim. Indeed, our understanding of the movement of the bullet is determined entirely by our knowledge of links in the chain of cause and effect, not by any direct observation that has ever been made of a bullet in flight. We might be tempted to think that bullets have been photographed in flight, but this is not true, of course; our photographs are a series of still frames, and we must trust in logical inference and perceptual illusion if we are to believe that there is movement between the frames of the motion picture or the sweeps of a television cathode-ray tube. Our knowledge of the causal chain of events is a 
series of still frames; we "know" links in the chain of events, and we make assumptions about the transitions from link to link. But in analyzing the chain of events between the squeezing of the trigger and the falling of the victim, it would certainly seem foolish to suggest that the "skin" of the gun should be an appropriate boundary between the causal events we are permitted to analyze and the causal events that belong in the domain of an off-limits lower level of analysis. In this case, we do not foolishly designate the trigger as part of the domain of behavior and the hammer as part of the domain of physiology, and suggest that there is some logical reason not to wonder as to the relations between the two.

Therefore, I take serious issue with Blackburn and Pfaus's argument that physiology is an inappropriate level of analysis. The argument that behavior and physiology represent different levels of analysis rather than nested and arbitrary conceptual categories reflects what Gilbert Ryle (1949) called a "category error," and it is time for psychologists to put it behind them. The practical difficulties involved in studying the teachings of two disciplines constitute a sound reason (though not a very noble one) not to attempt it, but the attempt to rationalize our resistance to interdisciplinary thinking does not.

My attempt to define motivation in terms of modulation does not, in any case, rest only on neurophysiological fact (or fiction). In my paper the notion of modulation is discussed in terms of behavioral as well as neurophysiological facts. A brief perusal of the citation record of the work of Komasaruk, Lehrman, Pfaff, Flynn and their colleagues-to choose a few examples that were carefully discussed in my paper-makes it clear that behavioral evidence of modulation is taken quite seriously in the scientific community. The hypothalamic stimulation or hormonal manipulations of these workers-all of which are analyzed and some of which are manipulated well within the domain of behavior-do not exert the same quality of behavioral control as is exerted by the tactile probing of the lip of the cat or the stroking of the flanks of the estrous rat. It is this point that is central to my attempt to define motivational phenomena as distinct from Pavlov's or Skinner's stimuli.

Questions of level of analysis aside, Blackburn and Pfaus are concerned that the notion of modulation may be as ephemeral as the notion of motivation. This is a serious concern; as our knowledge becomes more detailed it may prove as difficult to distinguish neuromodulators from classic neurotransmitters as it has been to distinguish motivational phenomena from nonmotivational phenomena. If Blackburn and Pfaus are right about this, then the definition will prove unworkable and soon be set aside.

I have tried to capture in the term "modulation" what I think to be the central notion in all workable theories of motivation. Blackburn and Pfaus close their comments with the statement that they "do not agree that motivation is a state in which responsiveness is modulated." It is difficult for me to accept that they literally mean what they have written; I can't imagine that they would contest the suggestion that responsiveness to food is greater when we are hungry and less when we are sated. I believe that this suggestion-fundamental to the distinction I offer between motivational and nonmotivational influences on behavior-is consistent with the thinking of most motivational theorists. Blackburn and Pfaus, in an earlier draft of their comment, suggested that motivation is a construct "to account for variations in the responses elicited by constant stimuli"; this is certainly the accepted view in ethology. Whatever the view of other motivational specialists on my formulation, I see the alternative suggested by Blackburn and Pfaus-that motivation is "the study of why animals do what they do when they do it"as a prototypical example of the kind of definition they say Skinner and I have "so cogently criticized." It is just what must be avoided: a construct that can only be inferred from the very behavior that it is used to explain. Whatever our final definition of motivation, it is an empty construct if it fails to meet this criticism.

In my view, the concept of motivation is theoretically useful if and only if it helps us to separate the factors that exert behavioral control into mutually exclusive categories. As I attempted to make clear in my article, I see the category of motivational variables as being clearly distinct from the category of stimulus or "releaser" variables. If we can establish it as an independent and objectively defined category of controlling variable, I see motivation as a useful construct. For Blackburn and Pfaus, motivation is a concept that cannot (if we are to take their closing sentence at face value) even be distinguished from the primal force of life itself. If it is merely to be a synonym for the sum of behavioral control mechanisms, I would have to agree that it is the empty and redundant label that Skinner has asserted it to be.

The task for Blackburn and Pfaus, if they do not like the formulation I have offered, is to suggest an alternative that does not regress motivational theory to the circularity that Skinner detected in older views. Blackburn and Pfaus's suggestion does not meet this criterion and does not satisfy any real need in psychological theory; they offer us a millstone rather than a milestone. We already have two perfectly good words to describe "the study of why animals do what they do when they do it": behaviorism and ethology. Indeed, it is unfortunate that we have even two words for this single referent, because the existence of the different words contributes to the illusion of different subject matter and adds to the distance between two groups of workers with common stated interests. Surely we need no third group of putative specialists claiming the same all-encompassing subject matter.

\section{REFERENCES}

Blackburn, J.R., \& Pfaus, J. G. (1988). Is motivation really modulation? A comment on Wise. Psychobiology, 16, 303-304.

RYLE, G. (1949). The concept of mind. London: Hutchinson.

WISE, R. A. (1987). Sensorimotor modulation and the variable action pattern (VAP): Toward a noncircular definition of drive and motivation. Psychobiology, 15, 7-20.

(Manuscript received April 13, 1988; accepted for publication April 21, 1988.) 